\title{
SOME RESULTS ON THE CENTER OF A RING WITH POLYNOMIAL IDENTITY
}

\author{
BY LOUIS ROWEN \\ Communicated by Nathan Jacobson, August 10, 1972
}

Introduction. The purpose of this paper is to provide a fresh outlook to various questions on rings with polynomial identity by examining the centers of such rings. This approach yields the interesting result that any nonzero ideal of a semiprime ring with polynomial identity intersects the center nontrivially (Theorem 2).

There are at least two interesting consequences to Theorem 2: a generalization of Wedderburn's theorem (any semiprimitive ring with polynomial identity, whose center is a field, is simple) and a strengthening of Posner's theorem [1] (any prime ring with a polynomial identity has a simple ring of quotients whose center is the quotient field of the center of the prime ring).

The proofs are elementary modulo Jacobson [3]. Of course rings are not necessarily commutative and for the sake of simplicity we assume a unit 1 .

The key argument in this paper is an application of Formanek's central polynomials for matrix algebras over a field, whose important properties are [2]: Let $M_{n}$ be an $n \times n$ matrix algebra over an arbitrary field. Then there exists a polynomial $g_{n}\left(X_{1}, \ldots, X_{m}\right)$ which has coefficients in $Z$; is homogeneous (degree $>0$ ) in every variable and linear in all but the first variable; takes values in the center for every specialization in $M_{n}$; and is nonvanishing for some specialization.

LEMma 1. $g_{n}\left(X_{1}, \ldots, X_{m}\right)$ is central, nonvanishing for any central simple algebra $S$ of degree $n$ over its center $C$.

Proof. Let us first consider $C$ finite. Since by Wedderburn's structure theorem $S$ is a matrix algebra over a division ring $D$ which is finite dimensional over $C$, which is finite, we have $D$ is finite and thus a field (Wedderburn's theorem on finite division rings [3, p. 183]). Thus $D=C$ and $S$ is in fact a matrix algebra over $C$, a field, and $g_{n}$ is by hypothesis a central, nonvanishing polynomial for $S$, so that there is nothing to prove.

So we may assume $C$ is infinite. Again let $S$ be a matrix algebra over $D$, a division ring finite dimensional over $C$. Let $F$ be a splitting subfield

AMS (MOS) subject classifications (1970). Primary 16A08, 16A12, 16A20, 16A38; Secondary 16A02, 16A18, 16A40, 16A88.

Key words and phrases. Central polynomials, ideals of center, polynomial identity, prime ring, ring of quotients, semiprime ring, semiprimitive ring, semisimple ring. 
of $D\left[3\right.$, p. 120]. Then $S \otimes_{C} F=M_{n}(F)$, for which $g_{n}$ is central and nonvanishing, so $g_{n} X_{m+1}-X_{m+1} g_{n}$ is a polynomial identity for $S$ (since $C$ is a field, $S \hookrightarrow S \otimes_{C} F$ by $s \rightarrow s \otimes 1$, so $g_{n} X_{m+1}-X_{m+1} g_{n}$, which vanishes on $M_{n}(F)$, vanishes on the subalgebra $\left.S\right)$. Thus, $g_{n}$ takes values in the center of $S$. Is $g_{n}$ nonvanishing? If $g_{n}$ were a polynomial identity for $S$, it would be a polynomial identity for $M_{n}(F)=S \otimes_{C} F$ since $C$ is infinite [3, p. 231], so $g_{n}$ would vanish for all specializations in $M_{n}(F)$, false. So $g_{n}$ does not vanish for some specializations in $S$, and we are done.

THEOREM 1. Let $R$ be semiprimitive (= semisimple in the sense of Jacobson [3]) with center $C$ and polynomial identity $f$. Let $A$ be any ideal $\neq 0$ of $R$. Then $A \cap C \neq 0$.

Proof. As a semiprimitive ring, $R$ is a subdirect product of primitive rings $S_{i}$, each of which is a homomorphic image of $R$. Thus, $S_{i}$ satisfies the same polynomial identity $f$ and is therefore simple of dimension $\leqq[d / 2]^{2}$ over its center, which we shall call $C_{i}$ (by Kaplansky's Theorem, $[3$, p. 226]).

Since $\bigcap_{i} \operatorname{ker} p_{i}=0$ where $p_{i}$ is the canonical projection $p_{i}: R \rightarrow S_{i}$, we must have the restriction of $p_{i}$ to $A$ is nonzero for some $i$. Let $I=\left\{i: p_{i}\right.$ restricted to $A$ is nonzero $\}$, i.e. for $i \notin I$ the $i$ th component of any element of $A$ is 0 . Since $p_{i}: R \rightarrow S_{i}$ is surjective, $p_{i}(A)$ is a nonzero ideal of $S_{i}$ for $i \in I$. But $S_{i}$ is simple. Therefore, $\forall i \in I, p_{i}$ restricted to $A$ is surjective.

Now let us choose $i_{0} \in I$ such that $S_{i_{0}}$ has maximal dimension $n_{0}^{2}$ over its center $\forall i \in I$ (the dimension is bounded by $[d / 2]^{2}$ by Kaplansky's theorem [3, p. 226]). Then let $g_{n_{0}}$ be the nonvanishing central polynomial of Formanek, described in Lemma 1. Since $g_{n_{0}}$ is central for $M_{n_{0}}, g_{n_{0}}$ is central (Procesi has shown indeed vanishing) for smaller-degree matrix algebras, hence for all smaller-degree central simple algebras, by the argument of Lemma 1. So $g_{n_{0}}$ is central for $S_{i}, \forall i \in I$.

Let $s_{1}, \ldots, s_{m} \in S_{i_{0}}$ such that $g_{n_{0}}\left(s_{1}, \ldots, s_{m}\right) \neq 0$. Let $a_{1}, \ldots, a_{m} \in A$ such that $p_{i_{0}}\left(a_{j}\right)=s_{j}, 1 \leqq j \leqq m$. Since $g_{n_{0}}$ is homogeneous of degree $>0$, its constant term is 0 , and

$$
\begin{array}{ll}
\forall i \notin I, & p_{i}\left(g_{n_{0}}\left(a_{1}, \ldots, a_{m}\right)\right)=g_{n_{0}}\left(p_{i}\left(a_{1}\right), \ldots, p_{i}\left(a_{m}\right)\right)=g_{n_{0}}(0, \ldots, 0)=0 . \\
\forall i \in I, & p_{i}\left(g_{n_{0}}\left(a_{1}, \ldots, a_{m}\right)\right)=g_{n_{0}}\left(p_{i}\left(a_{1}\right), \ldots, p_{i}\left(a_{m}\right)\right) \in C_{i}
\end{array}
$$

and in particular

$$
\begin{aligned}
p_{i_{0}}\left(g_{n_{0}}\left(a_{1}, \ldots, a_{m}\right)\right) & =g_{n_{0}}\left(p_{i_{0}}\left(a_{1}\right), \ldots, p_{i_{0}}\left(a_{m}\right)\right) \\
& =g_{n_{0}}\left(s_{1}, \ldots, s_{m}\right) \neq 0 \quad \text { but } \in C_{i_{0}} .
\end{aligned}
$$


So $g_{n_{0}}\left(a_{1}, \ldots, a_{m}\right) \in C$ and is nonzero. But $g_{n_{0}}\left(a_{1}, \ldots, a_{m}\right) \in A$, so $A \cap C \neq 0$. Q.E.D.

COROLlaRY. Let $R$ be semiprimitive with center $C$, a field, and with polynomial identity $f$. Then $R$ is simple.

Proof. If $A$ is a nonzero ideal of $R, A \cap C \neq 0$ by Theorem 1 . But $C$ is a field, so $A=R$. Q.E.D.

It is clear that the conditions of the corollary are necessary; appropriate examples of their necessity are the nonsimple rings $\boldsymbol{Z}$, the ring of integers (center is not a field), and the ring of linear transformations over an infinite-dimensional vector space over a division ring (primitive, center is a field, but has no polynomial identity).

We now generalize Theorem 1:

THEOREM 2. Let $R$ be semiprime with center $C$ and polynomial identity $f$. Let $A$ be any ideal $\neq 0$ of $R$. Then $A \cap C \neq 0$.

Proof. Since a semiprime ring with polynomial identity has no nil ideals (an immediate consequence of [3, p. 232, Theorem 1]), $R[\lambda]$ is semiprimitive [3, p. 12], where $R[\lambda]$ is the ring of polynomials in the commuting indeterminate $\lambda$, with coefficients in $R$ (these reductions have been made standard by Amitsur).

We note that the center of $R[\lambda]$ is actually $C[\lambda]$, since if $c(\lambda)=\sum r_{i} \lambda^{i}$, $r_{i} \in R$, then $\forall r \in R, c(\lambda) r=r c(\lambda) \Rightarrow \sum\left(r_{i} r-r r_{i}\right) \lambda^{i}=0 \Rightarrow r_{i} \in C$, so $c(\lambda) \in$ $C[\lambda]$.

Now $A$ is an ideal of $R$, so $A[\lambda]$ is an ideal of $R[\lambda]$. $R[\lambda]$ is semiprimitive, so by Theorem $1 A[\lambda] \cap C[\lambda] \neq 0$. Comparing coefficients we get $A \cap C$ $\neq 0$. Q.E.D.

COROLlaRY 1. (Strengthening of Posner's theorem). Let $R$ be a prime ring with center $C$ and polynomial identity. Then there exists a simple ring $S=R \widetilde{C}$, where $\tilde{C}$ is the quotient field of $C$, which is a ring of quotients of $R$ and is finite dimensional over $\widetilde{C}$ (this is a statement of Posner's theorem [1]). Moreover, the center of $S$ is $\widetilde{C}$ (not part of the previous formulations of Posner's theorem).

Proof. Since $R$ is a prime, its center $C$ has no zero-divisors. So we define $S$ formally as $\left\{r c^{-1}, r \in R, c \neq 0 \in C \mid r_{1} c_{1}^{-1}=r_{2} c_{2}^{-1}\right.$ if and only if $r_{1} c_{2}$ $\left.=r_{2} c_{1}\right\}$. Then multiplication and addition are set in the obvious ways:

$$
\left(r_{1} c_{1}^{-1}\right)\left(r_{2} c_{2}^{-1}\right)=\left(r_{1} r_{2}\right)\left(c_{1} c_{2}\right)^{-1}
$$

and 


$$
r_{1} c_{1}^{-1}+r_{2} c_{2}^{-1}=\left(r_{1} c_{1}+r_{2} c_{1}\right)\left(c_{1} c_{2}\right)^{-1} .
$$

All the necessary properties of multiplication and addition in $S$ are natural and immediate. Obviously $S=R \widetilde{C}$, and we claim $\widetilde{C}=$ cent $S$ :

It is obvious that $\widetilde{C} \subseteq$ cent $S$. To go the other way, let $r c^{-1} \in \operatorname{cent} S$. Then $\left(r c^{-1}\right) c \in$ cent $S$, so $r \in R \cap$ cent $S \subseteq C$. So $r c^{-1} \in \widetilde{C}$ and cent $S=\widetilde{C}$, a field.

Clearly $S$ is a prime: Suppose $s_{1} S s_{2}=0, s_{1}, s_{2} \in S$. Then $s_{1}\left(r c^{-1}\right) s=0$, $\forall r \in R, c \neq 0 \in C$. Let $s_{1}=r_{1} c_{1}^{-1}, s_{2}=r_{2} c_{2}^{-1}, r_{i} \in R, i=1,2, c_{i} \in C$, $i=1,2$. Then $0=r_{1} c_{1}^{-1} r c^{-1} r_{2} c_{2}^{-1}=\left(r_{1} r r_{2}\right)\left(c_{2} c c_{1}\right)^{-1}, \forall r \in R, c \in C$. So $r_{1} r r_{2}=0, \forall r \in R$. But $R$ is prime, so either $r_{1}$ or $r_{2}$ is 0 , so either $s_{1}$ or $s_{2}$ is 0 .

So $S$ is prime and $S=R \widetilde{C}$ satisfies a polynomial identity. Therefore by Theorem 2, if $A$ is a nonzero ideal of $S, A \cap \widetilde{C} \neq 0$. But $\widetilde{C}$ is a field, so $A=S$ and $S$ is simple. $S$ is finite dimensional over $\widetilde{C}$ by Kaplansky's theorem. Q.E.D.

I am deeply indebted to Professor Jacobson for his patient guidance.

ADDENDUM. Subsequent to the submission of this note for publication, several facts have come to my attention. The most significant fact is that the statement of Corollary 1 of Theorem 2 (the strengthening of Posner's theorem) has been discovered also by Procesi, Formanek, Martindale, and Small, so this result cannot be considered new. What is new is the straightforward and direct proof, relying only on the existence of central polynomials for matrix rings. Using the stronger version of Posner's theorem, Small (and perhaps others) proved the special case of Theorem 2 for $R$ prime.

Martindale has observed to me that the results of this paper remain true even if one does not assume $R$ contains unity; this assumption is not essential to any of the proofs. At any rate, one could always adjoin unity to a semiprime ring with polynomial identity to obtain a semiprime ring with polynomial identity and with unity, thereby extending Theorem 2 to the analogous result for rings without unity. It follows clearly that any semiprime ring with polynomial identity has a nontrivial center. That semisimple rings with polynomial identity have nontrivial centers, was first shown by Procesi (remark 5 of [2]). Using standard methods of embedding semiprime rings with polynomial identity into semisimple rings with polynomial identity (as indicated in the proof of Theorem 2), can one extend Procesi's result to semiprime rings with polynomial identity, as was observed by Small. This result is much weaker than Theorem 2, however, because Theorem 2 fails for rings which satisfy precisely the same polynomial identities as $n \times n$ matrix rings; it is easy to see that these rings have nontrivial centers. 


\section{REFERENCES}

1. S. A. Amitsur, Prime rings having polynomial identities with arbitrary coefficients, Proc. London Math. Soc. (3) 17 (1967), 470-486. MR 36 \# 209.

2. E. Formanek, Central polynomials for matrix rings, J. Algebra 23 (1972).

3. N. Jacobson, Structure of rings, Amer. Math. Soc. Colloq. Publ., vol. 37, Amer. Math. Soc., Providence, R.I., 1964. MR 36 \# 5158.

4. C. Procesi, Non-commutative affine rings, Atti Accad. Naz. Lincei Mem. C1. Sci. Fis. Mat. Natur. Sez. I (8) 8 (1967), 237-255. MR 37 \# 256.

Department of Mathematics, Yale University, New Haven, Connecticut 06520 